Arch. Tierz., Dummerstorf 44 (2001) 1, 71-88

Aus dem Institut fur Tierzucht und Tierhaltung mit Tierklinik der Martin-Luther Universital Halle-Wittenberg

HENDRIK HARANT, RALF PORZIG, JENS THIELEBEIN, WERNER SAAR

und LUTZ SCHÖLER

\title{
Gewichtsentwicklung beim Kaninchen (Oryctolagus cuniculus) nach Transfer reziproker Kernkombinationen in eine neutrale Ammenlinie
}

\begin{abstract}
Summary
Title of the paper: Development of body weight in rabbits (Oryctolagus cunlculas) after embryo transfer of reciprocal nuclear combinations to a neutral line of recipients.

The article describes differences in the development of body weight of the Fl-generations in the rabbit, which occured after embryo transfer from reciprocal nuclear combinations to a neutral line of recipients. These differences can be explained as expression of maternal cyto-plasmatical and / or imprinting-effects. The two races - Deutsche Riesen and Farbzwerge -, which are extremly different in body weight were used for the reciprocal crosses. The neutral recipients were a commercial breed „Zika“. Results show a significant higher litter weight of the offspring which descended from the heavier mother race. On the 84th day of life exists no differences in weight between the crosses. Differences were within the crosses between the females and males and between the crosses reciprocal different. Causes for the reciprocal sexdimorphism can be $A$ : extrachromosomal respectively cytoplasmatic factors or B: a sex-linked and a sex-influenced heredity, which coinside and are regulated by imprinting.
\end{abstract}

Key Words: rabbit, reciprocal cross, embryo transfer, body weight, maternal cytoplasmic effects, imprinting, sexlinked heredity

\section{Zusammenfassung}

Es werden phänotypische Merkmalsdifferenzen der F1-Generationen beim Kaninchen (Oryctolagus cuniculus) dargestellt, die nach chirurgischen Eileitertransfers von reziproken Kernkombinationen auf eine neutrale Ammenlinie auftraten und Ausdruck von maternal zytoplasmatischen und / oder Imprinting-Effekten scin könnten. Die beiden hinsichtlich ihrer Lebendmasse extrem unterschiedlichen Rassen - Deutsche Riesen und Farbzwerge - wurden als Kreuzungspartner genutzt, Als Ammen fungierten Zimmermannkaninchen. Die Ergebnisse zeigen ein statistisch signifikant höheres Wurfgewicht derjenigen Nachkommen, deren Mütter der größeren bzw. schwereren Rasse angehठ̈rten. Am 84. Lebenstag existieren zwischen den reziproken Nachkommengruppen keine Gewichtsunterschiede. Diese zeigten sich innerhalb der Kreuzungen zwischen den Geschlechtern und zwischen den Kreuzungen reziprok verschieden. Ursachen fur den wechselseitigen Geschlechtsdimorphismus können A: extrachromosomale bzw, zytoplasmatische Faktoren oder B: eine geschlechtsgebundenc und eine geschlechtsbeeinflußte Vererbung sein, weiche gemeinsam auftreten und durch Imprinting geregelt werden.

Schlusselwörter: Kaninchen, reziproke Kreuzung, Embryotransfer, Körpergewicht, maternal zytoplasmatische Effekte, Imprinting, geschlechtsgebundene Vererbung

\section{Einleitung}

Untersuchungen an Nachkommen reziproker Kreuzungen, bei denen die Elterntiere sich hinsichtlich ihrer Körpergröße stark voneinander unterschieden, führten zu dem Ergebnis, daß die F1- Generationen jeweils eine größere Ähnlichkeit zu dem Phänotyp des Muttertieres aufwiesen. Die wohl bekanntesten Beispiele hierfür sind die reziproken Kreuzungen zwischen Pferd und Esel bzw. zwischen Shire horse und Shetland 
pony. Das aus Pferdestute $x$ Eselhengst hervorgegangene Maultier und der aus Eselstute x Pferdehengst hervorgegangene Maulesel ähnelte jeweils dem Erscheinungsbild der Mutter. Die Größe der geborenen Fohlen nach reziproker Verpaarung von Shire horse $\mathrm{x}$ Shetland pony richtete sich in beiden Fällen nach der Größe der Mutter. Während des weiteren Wachstums beider Kombinationen verringerten sich diese Unterschiede, aber es kam nicht zu einem vollkommenen Größenausgleich (WALTON und HAMMOND, 1938), Extreme Rassenkreuzungen beim Kaninchen (VENGE, 1953; JOHANSSON und VENGE, 1953; STENGEL, 1962), Rind (JOUBERT und HAMMOND, 1954) und Schwein (HARING et al., 1966) ergaben ein höheres Körpergewicht derjenigen Nachkommen, deren Mütter der größeren bzw. schwereren Rasse angehörten.

In den angeführten Fällen stellt jedoch der prä- und postnatale Umwelteinfluß der Mutter auf die Nachkommen eine entscheidende und nicht zu vernachlässigende Größe dar. VENGE (1950) transferierte befruchtete Eier der kleinen Hermelinrasse auf Blaue Wiener oder Belgische Riesen und von diesen großen Rassen auf die kleine Rasse. Durch das optimale uterine Milieu für die Hermelinföten vergrößerte sich ihr Geburtsgewicht, das der großen Rassen verringerte sich. Auch in der postnatalen Periode übten die unterschiedlichen Bedingungen einen bleibenden Einfluß aus. So erreichten die durch Transplantation auf die jeweilige größere bzw. kleinere Rasse geborenen Jungtiere nicht das Endgewicht ihrer normal geborener Rassen, wobei das Gewicht der kleinen übertragenen Rasse nicht wesentlich vom „Normalgewicht" differierte (VENGE, 1953).

Die intra-uterine Umwelt, welche wiederum von der Größe, dem Alter und dem Ernährungszustand abhängt, sowie das Aufzuchtvermögen der Mutter nach der Geburt üben auf das Wachstum junger Tiere wesentlichen Einfluß aus (HAFEZ, 1963).

Gefundene Wachstumsunterschiede nach reziproken Kreuzungen müssen aber nicht unbedingt nur Ausdruck des maternalen Umwelteinflusses sein. Als Ursache der phänotypischen Varianz könnten ebenso extrachromosomale Vererbung und / oder Genetisches Imprinting in Frage kommen.

Extrachromosomale Vererbung bezieht sich auf die monoparentale Vererbung der auBerhalb des Zellkerns lokalisierten DNA der Mitochondrien (mtDNA). Der wahrscheinlich furr alle Wirbeltiere typisch rein maternale Vererbungsmodus dieser Organell-DNA wurde für Amphibien durch DAWID und BLACKLER (1972) belegt. 1974 wiesen HUTCHISON et al. nach, daß die aus den Haustieren Pferd und Esel erzeugten reziproken Artbastarde nur das fur die als Mutter eingesetzte Art typische DNA-Fragmentmuster in ihren Mitochondrien aufwiesen. DE GEORGI und SACCONE (1989) sowie WOLSTENHOLME (1992) bewiesen, daß bei Vertebraten innerhalb einer Art das mitochondriale Genom rein maternal vererbt wird und Rekombinationsmechanismen fehlen. WEHNER und GERING berichteten 1990, daß die maternale Vererbung der mtDNA für Menschen, Xenopus und Drosophila bestätigt wurde. Zytoplasmatische Einflusse, d.h. Einflüsse von mtDNA auf den Phänotyp der Nachkommen, werden damit immer maternal vererbt.

Im Gegensatz zu dem den generellen biparentalen Vererbungsmodus betreffenden Fall der mtDNA, wird beim Genetischen Imprinting die unterschiedliche Expression und 
phänotypische Wirksamkeit eines bestimmten, umfangmäßig noch nicht quantifizierbaren Anteils der Kern-gebundenen Gene in Abhängigkeit von ihrer paternalen oder maternalen Herkunft verstanden. Durch den Zusatz unterschiedlicher Adjektive wird versucht, die jeweilige Ebene und den Umfang der betroffenen genetischen Informationsbestände näher zu beschreiben (genomisches-, chromosomales-, Allel-spezifisches-, Linien- oder Spezies-spezifisches Imprinting). Das Phänomen des Imprintings wurde zuerst bei Insekten (SCHRADER,1921; SCHRADER und HUGHES-SCHRADER, 1931; HUGHES-SCHRADER, 1935; HUGHES-SCHRADER, 1948; CHANDRA und BROWN, 1975; NUR, 1990), später bei Säugetieren (COOPER et al., 1971; RICHARDSON et al., 1971; TAKAGI und SASAKI, 1975), Hefepilzen (KL.AR, 1990) und höheren Pflanzen (KERMICLE und ALLEMAN, 1990; FLAVELL und O'DELL, 1990; HESLOP-HARRISON, 1990) entdeckt und verdeutlichte den großen Einfluß dieses Phänomens auf diverse Entwicklungsvorgänge.

Die nach konventionellen Kreuzungen auftretenden reziproken Merkmalsdifferenzen können somit als Summe von maternal uterinen und postnatalen Einflußgrößen sowie von möglichen maternal-zytoplasmatischen- und Imprinting-Effekten verstanden werden. Aufgrund der Beeinflußung und Überlagerung untereinander ist die Deutung der Wirkung jedes einzelnen Summanden aber stark begrenzt. Nur durch Neutralisation der einzelnen Einflußkomplexe ist ihre Beurteilung möglich. Mit Hilfe der heute zur Verfügung stehenden Methoden des experimentellen Eingriffs in die embryonale Frühentwicklung kann dieses Ziel erreicht werden. Die Abgrenzung der maternalen prä- bzw, postnatalen Effekte von maternal zytoplasmatischen- und Imprintingeffekten ist durch den Embryotransfer der reziprok erzeugten Nachkommen auf eine „neutrale“ Ammenlinie möglich. Der Einfluß der maternalen Umweltfaktoren auf die reziproken Kernkombinationen (KK) kann dadurch konstant gehalten werden. Auftretende Merkmalsdifferenzen unter den Nachkommen können als Ausdruck maternal zytoplasmatischer- und / oder Imprinting-Effekte angesehen werden. Ihre spätere Separation in Effekte des Zytoplasmas und die des Zellkernes kann durch die Einbringung reziproker Zellkerne in entkernte Eizellen mit definiertem Zytoplasma mittels mikrochirurgischer Kerntransplantation bzw. Elektrozellfusion genannter Eizellen mit Furchungszellen (Blastomeren) aus Embryonen entsprechender reziproker Kernkombinationen geschehen.

Im Hinblick auf die mikrochirurgische Manipulation ergibt sich folgende Fragestellung. Existieren nach dem Transfer reziproker Kernkombinationen auf eine neutrale Ammenlinie beim Kaninchen Merkmalsdifferenzen zwischen den Phänotypen, die Ausdruck von maternal zytoplasmatischen und / oder Imprinting-Effekten sein können? Aufgrund bisheriger Erkenntnisse dürften derartige Effekte vorwiegend in den Prozeßverläufen der Differenzierung, Morphogenese, Vitalität/Letalität und Entwicklungsintensităt auftreten und somit den Sektor der allgemeinen Leistungen wie Fertilität und prä- bzw. postnatales Wachstum berühren. Die Bearbeitung der Fragestellung erfolgte an stark in ihrem Körpergewicht divergierenden Kaninchenlinien bzw. -rassen, aus denen die reziproken Kreuzungen hervorgingen. Als Ammenlinie fanden ,normalgroße" Tiere Verwendung. 
HARANT u.a: Gewichtsentwicklung beim Kaninehen nach Transfer reziproker Kernkombinationen in eine neutrale Ammenlinie

\section{Material und Methoden}

Für die Untersuchungen zur Bearbeitung der Fragestellung standen Häsinnen und Rammler der Rassen Deutsche Riesen (DR [grau]) und Farbzwerge (FBZW [Russenfärbung; grau; rot]) sowie Häsinnen des Zuchtproduktes Zimmermannkaninchen (Zika) zur Verfügung. Die Tiere wurden in Flatdecks in einem klimatisierten Stall mit gleichbleibendem Lichtregime gehalten. Aufgrund der Körpergröße der DR erfolgte ihre Aufstallung ab 84. Lebenstag in größeren Einzelkäfigen bzw. in Bodenabteilen mit Einstreu.

Die beiden hinsichtlich ihrer Lebendmasse extrem unterschiedlichen Rassen DR und FBZW wurden als Kreuzungspartner für die reziproken Kreuzungen genutzt. Als Ammen fungierten die Zimmermannkaninchen, welche zum Zeitpunkt des Transfers ein Alter von 5 bis 6 Monaten und ein in etwa gleiches Körpergewicht aufwiesen sowie noch nicht zur Reproduktion herangezogen wurden. Zur Vermeidung von Scheinträchtigkeiten erfolgte eine Einzelaufstallung der Tiere 3 Wochen vor der Benutzung. Für die Erstellung der benötigten Kreuzungen (DR $\times$ FBZW bzw. FBZW $\times$ DR) wurde das Sperma der Böcke der jeweils anderen Rasse mittels künstlicher Besamung unverdünnt auf die Donoren übertragen. Die Ovulationsinduktion der künstlich besamten Spenderinnen und der Ammen wurde zeitgleich mit Gonavet bzw. Receptal $(0,2 \mathrm{ml})$ durchgeführt. Nach 15 - 27 h p.c. erfolgte die Schlachttötung der Spendertiere mit Betäubung durch Stirnschlag und anschließendem Ausbluten nach Durchtrennung der Halsschlagadern. Danach wurde die Bauchhöhle geöffnet und beidseitig die Ovarien, Eileiter und ein etwa $1 \mathrm{~cm}$ langes Stück der Uterusspitzen zusammenhängend entnommen. Darauffolgend geschah die Abpräparation der Ovarien- und Eileiterbänder, um eine leichtere Gewinnung der Embryonalstadien zu ermöglichen. Hierzu erfolgte die Spülung der gestreckten Eileiter. In das Infundibulum tubae wurde eine stumpf endende Kanüle eingeführt, von der die Spülflüssigkeit in Richtung der in einer Petrischale gelagerten Uterusspitze injiziert wurde. Die so aufgefangene Spülflüssigkeit (Dulbecco's PBS, $\mathrm{Mg}^{++}$- und $\mathrm{Ca}^{++}$-frei) enthielt die gewonnenen Embryonen. Nach Feststellung ihrer Anzahl und Beurteilung der Stadien begann die Zwischenkultur der befruchteten Eizellen bis zum Transfer auf die pseudograviden Ammen in EBSS (GIBCO BRL, $\mathrm{Mg}^{++}$- und $\mathrm{Ca}^{++}$-frei, ohne Phenol Rot) $+20 \%$ FKS bei $5 \% \mathrm{CO}_{2}, 37^{\circ} \mathrm{C}$ und $98 \%$ Lufteuchtigkeit in einem Incubator.

Die Übertragung der Embryonalstadien auf die Ammen fand mit chirurgischem Eileitertransfer statt. Nach Narkotisierung und Vorbereitung des Operationsfeldes erfolgte die Fixierung der Tiere. Ihre Bauchhöhle wurde mit einem 4-5 cm langen Schnitt entlang der Linea alba, zwischen Nabel und caudalstem Zitzenpaar, geöffnet. Der Transfer der Embryonen geschah nach Vorlagerung von Uterus, Eileiter und Ovar über das Infundibulum in den Isthmus. Im Anschluß, nach Rücklagerung der Organe, erfolgte der Verschluß der Bauchhöhle, indem das Peritoneum, die Muskelschichten sowie die Unterhaut vernäht wurden. Der Verschluß der Haut gelang durch Klammerung (Beiersdorf $\mathrm{AG}$, Leukoclip $\left.{ }^{\circledast} \mathrm{SD}\right)$. Die Klammern wurden 10 bis 12 Tage nach der Operation entfernt.

Die Embryonen der benötigten Kreuzungen sind im Rein- und im Gemischttransfer übertragen worden. Beim Reintransfer erfolgte die Übertragung einer reziproken Kreu- 
zung auf die Amme, beim Gemischttransfer wurden den Kreuzungen Ergänzungsembryonen (EE) hinzugefügt, um eine höhere übertragene Embryonenzahl (10) zu erreichen, die ADAMS (1962) als günstigste ansah. Als Ergänzungsembryonen fanden reine Zika-Embryonen des gleichen Entwicklungsstadiums Verwendung, welche durch natürlichen Deckakt oder künstliche Besamung erzeugt und in Art und Weise der Kreuzungsembryonen gewonnen wurden. Innerhalb eines Transfers fanden einmalig beide Kreuzungen Verwendung. Anhand differenter Fellfärbung konnten die geworfenen Jungtiere unterschieden werden.

Für die Bewertung und den Nachweis von Merkmalsdifferenzen der reziproken Kreuzungen, welche im Bereich von prä- und postnatalem Wachstum vermutet wurden, erfolgte die Gewichtsbestimmung zur Geburt und danach wöchentlich bis zum 84 . Lebenstag. Die Erfassung der Daten fand gleichfalls für die Ausgangsrassen (ohne Embryotransfer) statt. Das Körpergewicht zur Geburt und die weiteren Werte bis zum Ab. setzen vom Muttertier am 28, Lebenstag bestimmten sich aus dem Gesamtgewicht des Wurfes bezogen auf die einzelnen Nachkommen. Nach dem Absetzen ( $28 \mathrm{~d}$ ) erfolgte die Wägung individuell.

Die heranwachsenden Kaninchen erhielten bis zum 84. Lebenstag handelsübliches Kaninchenkraftfuiter (DEUKA) ad libitum, um das genetische Wachstumspotential voll auszuschöpfen. Natürlich und künstlich bedeckte Häsinnen sowie die Ammen bekamen $180-200 \mathrm{~g}$ dieses Futtermittels pro Tag. Wasser stand ad libitum zur Verfügung.

\section{Ergebnisse}

In den Tabellen 1-4 sind die Mittelwerte, die Standardabweichungen sowie die Stan* dardfehler der Mittelwerte der erfaßten Gewichte der reinen Rassen und ihrer reziproken Kreuzungen aufgeführt. Aufgrund des betrieblichen Ablaufes war eine lückenlose Erfassung der Gewichte nicht an allen Meßtagen möglich. Daraus resultieren die unterschiedlichen n-Zahlen,

Deutliche Unterschiede zu allen Bestimmungszeitpunkten ergaben sich zwischen den Elterntierrassen, deren Größenverhältnis im ausgewachsenem Zustand in etwa 1: 5 beträgt. Die Wachstumskurven der reziproken Kreuzungstiere liegen zwischen denen der reinen Rassen (Abb. 1) und uber dem Mittel beider. Sie unterscheiden sich nur im

\section{Tabelle 1}

Gewichtsentwicklung Deutsche Riesen in Abhángigkeit von Geschlecht und Alter (Development of weight Deutsche Riesen in dependence on sex and age) in $\mathrm{g}$

\begin{tabular}{|c|c|c|c|c|c|c|c|c|c|c|c|c|c|c|}
\hline \multicolumn{2}{|c|}{ Alter in Tagen } & 0 & 7 & 14 & 21 & 28 & 35 & 42 & 49 & 56 & 63 & 70 & 77 & 84 \\
\hline \multirow{4}{*}{ männlich } & \multirow{4}{*}{$\begin{array}{c}n \\
\text { MW } \\
\text { STAB } \\
\text { SMW }\end{array}$} & & & & & & 34 & 21 & 15 & 21 & 24 & 23 & 19 & 22 \\
\hline & & & & & & & 805,8 & 1275,3 & 1626,8 & 2119,6 & 2472,8 & 2947,4 & 3329,0 & 3685,6 \\
\hline & & & & & & & 172,2 & 172,7 & 158,2 & 221,1 & 303,4 & 393,5 & 394,2 & 520,0 \\
\hline & & & & & & & 29,5 & 37,7 & 40,8 & 48,2 & 61,9 & 82,1 & 90,4 & 110,9 \\
\hline \multirow{4}{*}{ weiblich } & \multirow{4}{*}{$\begin{array}{c}n \\
\text { MW } \\
\text { STAB } \\
\text { SMW }\end{array}$} & & & & & & 39 & 39 & 32 & 38 & 41 & 34 & 29 & 40 \\
\hline & & & & & & & 937,4 & 1304,0 & 1682,1 & 2160,0 & 2528,6 & 2964,4 & 3283,5 & 3645,6 \\
\hline & & & & & & & 339,4 & 301,3 & 361,7 & 350,0 & 428,3 & 487.7 & 531,8 & 457,1 \\
\hline & & & & & & & 54,4 & 48,2 & 63,9 & 56,8 & 66,9 & 83,7 & 98,8 & 72,3 \\
\hline \multirow{4}{*}{ gesamt. } & \multirow{4}{*}{$\begin{array}{c}n \\
\text { MW } \\
\text { STAB } \\
\text { SMW }\end{array}$} & 123 & 91 & 83 & 78 & 87 & 73 & 60 & 47 & 59 & 65 & 57 & 48 & 62 \\
\hline & & 78,9 & 154,0 & 290,2 & 433,0 & 584,4 & 876,2 & 1294,0 & 1664,4 & 2145,6 & 2508,0 & 2957,5 & 3301,5 & 3659,8 \\
\hline & & 24,5 & 59,0 & 88,3 & 115,9 & 148,9 & 280,6 & 262,0 & 310,6 & 308,8 & 385,3 & 448,4 & 478,0 & 476,5 \\
\hline & & 2,2 & 6,2 & 9,7 & 13,1 & 16,0 & 32,8 & 33,9 & 45,3 & 40,2 & 47,8 & 59,4 & 69,0 & 60,5 \\
\hline
\end{tabular}

n: Anzahl; MW: Mittelwert; STAB: Standardabweichung; SMW: Standardfehler des Mittelwertes 
Tabelle 2

Gewichtsentwicklung Farbzwerge (Russenfarbung) in Abhăngigkeit von Geschlecht und Alter (Development of weight Farbzwerge in dependence on sex and age) in $\mathrm{g}$

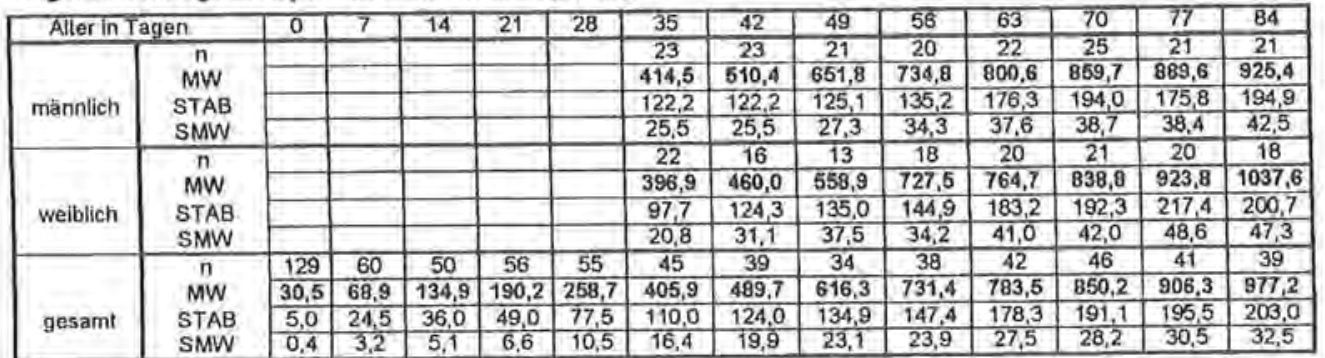

n: Anzahl; MW: Mittelwert; STAB: Standardabweichung: SMW: Standardfehfer des Mittelwerles

Tabelle 3

Gewichtsentwicklung F1 DR x FBZW nach Embryotransfer in Abhăngigkeit von Geschlecht und Alter (Development of weight FI DR x FBZW after embryo transfer in dependence on sex and age) in g

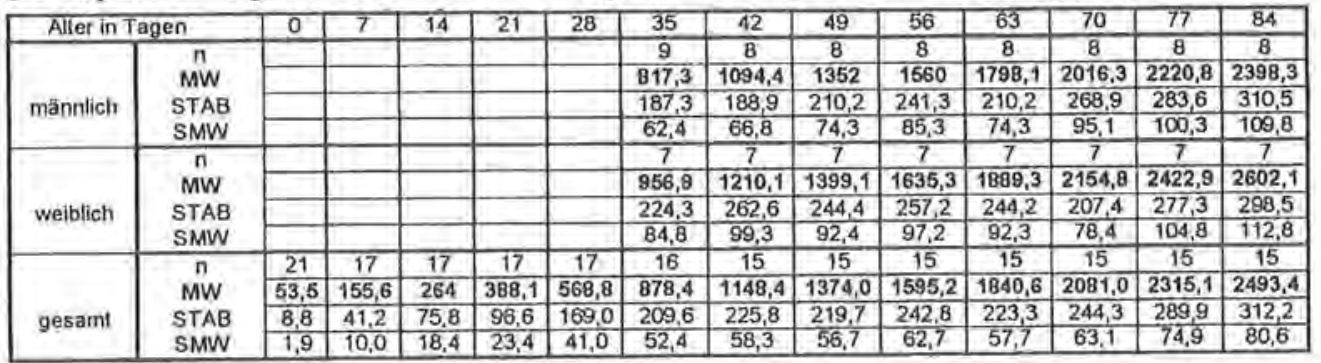

n: Anzahl; MW: Mittelwert; STAB: Standardabweichung; SMW: Standardfehler des Miltelwertes

Tabelle 4

Gewichtsentwicklung FL FBZW x DR nach Embryotransfer in Abhángigkeit von Geschlecht und Alter (Development of weight FI FBZW x DR after embryo iransfer in dependence on sex and age) in $g$

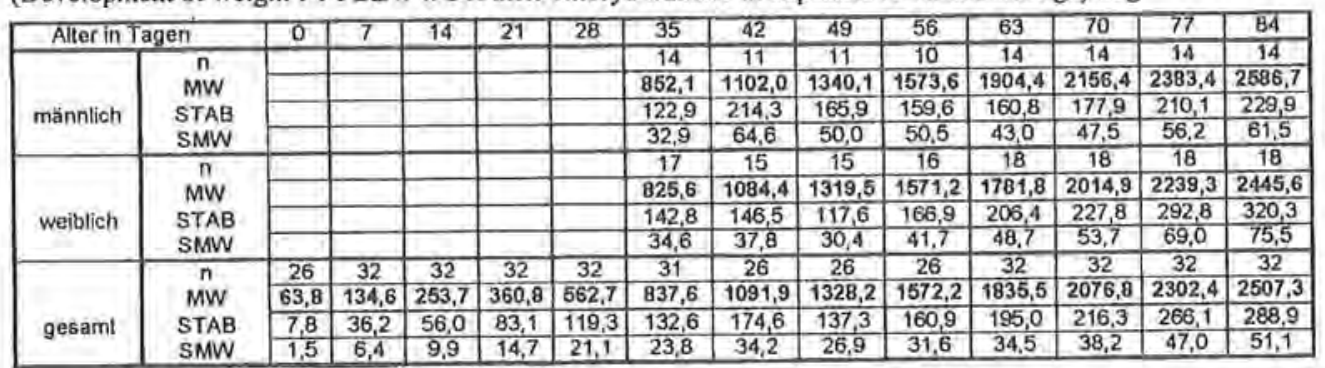

n: Anzaht; MW: Mittelwert; STAB: Standardabweichung; SMW: Standardfehler des Mittelwertes

Geburtsgewicht signifikant voneinander. Am 84. Lebenstag (LT) beträgt der Gewichtsunterschied der Mittelwerte der Kreuzungen 13,9 g, (0,56 \%; bezogen auf das Gewicht der leichteren Tiere).

Bei Betrachtung der Gewichtsentwicklung der Geschlechter zeigten sich Unterschiede in den reinen Rassen (Abb. 2; Abb, 3) und innerhalb beider Kreuzungen (Abb, 4, Abb. 5). Die männlichen DR besitzen am 84. Lebenstag ein um $40 \mathrm{~g}(1,09 \%)$, höheres 
Durchschnittsgewicht als ihre weiblichen Partner Die Farbzwergböcke sind zu diesem Zeitpunkt mit 112,2 g (12,12\%) Abweichung vom Mittelwert der weiblichen Tiere hinsichtlich des Körpergewichtes unterlegen.

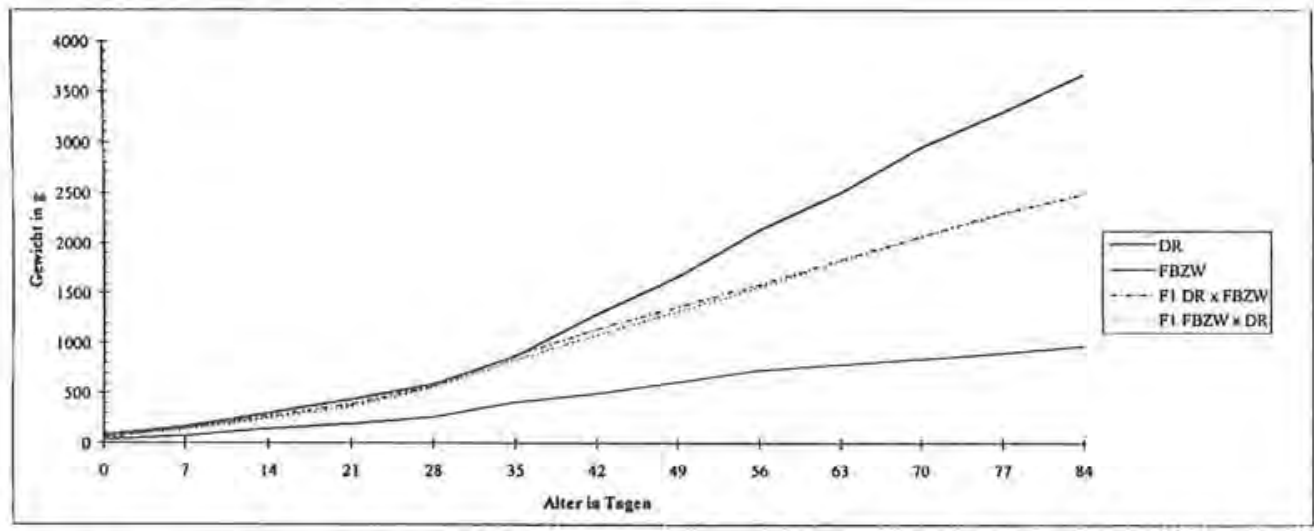

Abb. I: Gewichtsentwicklung der Elterntierrassen und ihrer reziproken Nachkommen (Development of weight of the parental races and their reciprocal descendants)

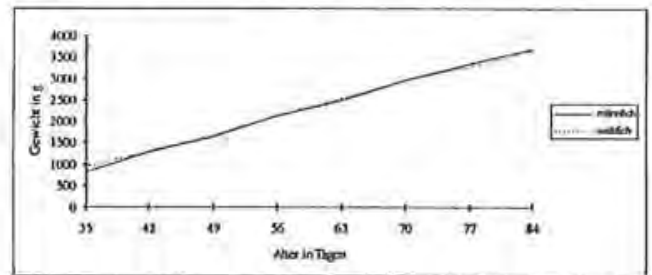

Abb. 2: Gewichtsentwicklung DR in Abhăngigkeit vom Geschlecht (Development of weight DR in dependence on sex)

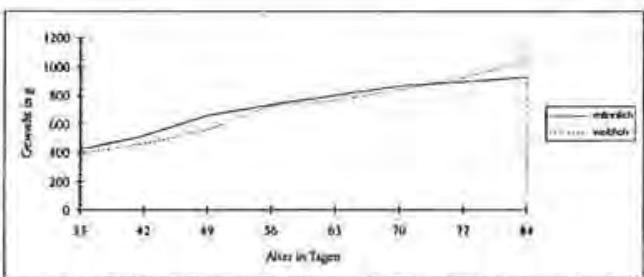

Abb, 3: Gewichtsentwicklung FBZW in Abhängigkeit vom Geschlecht (Development of weight FBZW in dependence on sex)

Die mănnlichen Nachkommen der Kreuzungsrichtung FBZW x DR bzw. die weiblichen der Paarung DR x FBZW wiesen, ebenfalls am 84. Lebenstag, ein größeres Gewicht als ihre weiblichen bzw. männlichen Vergleichstiere auf (Abb. 4; Abb. 5).

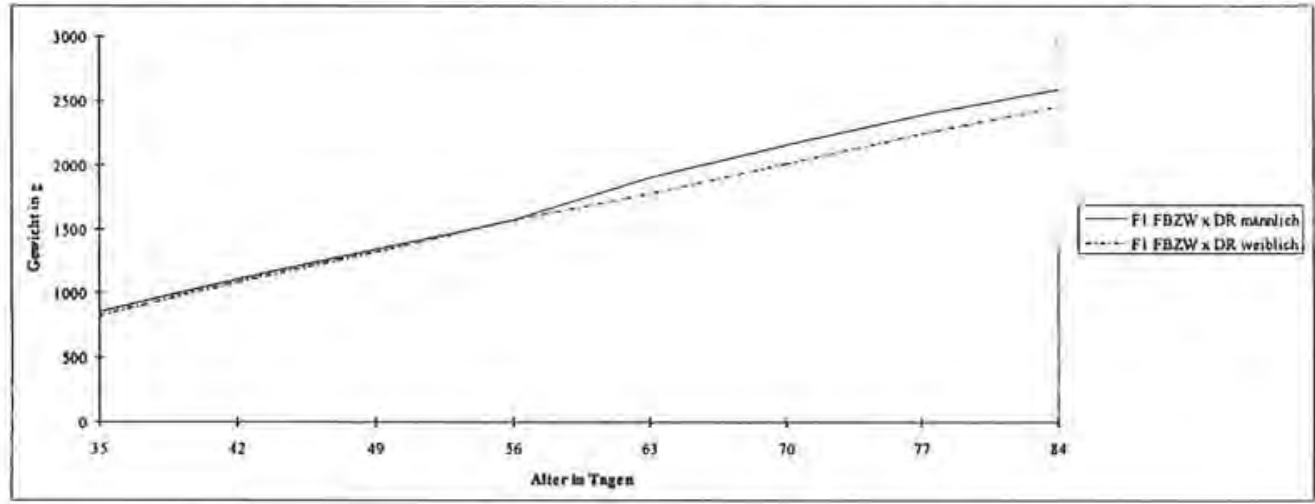

Abb. 4: Gewichtsentwicklung F1 FBZW x DR in Abhangigkeit vom Geschlecht (Development of weight F1 FBZW $x$ DR in dependence on sex) 
Dabei erreichten die männliche F1 FBZW x DR ein um 141,1 g $(5,76 \%)$ höheres durchschnittliches Gewicht als die weiblichen Tiere und die weibliche FI DR $x$ FBZW übertraf ihre männliche F1 um 203,8 g (8,49 \%). Die Verhältnisse zwischen den Geschlechtern ähneln in der Tendenz somit denen der jeweiligen Mutterrasse.

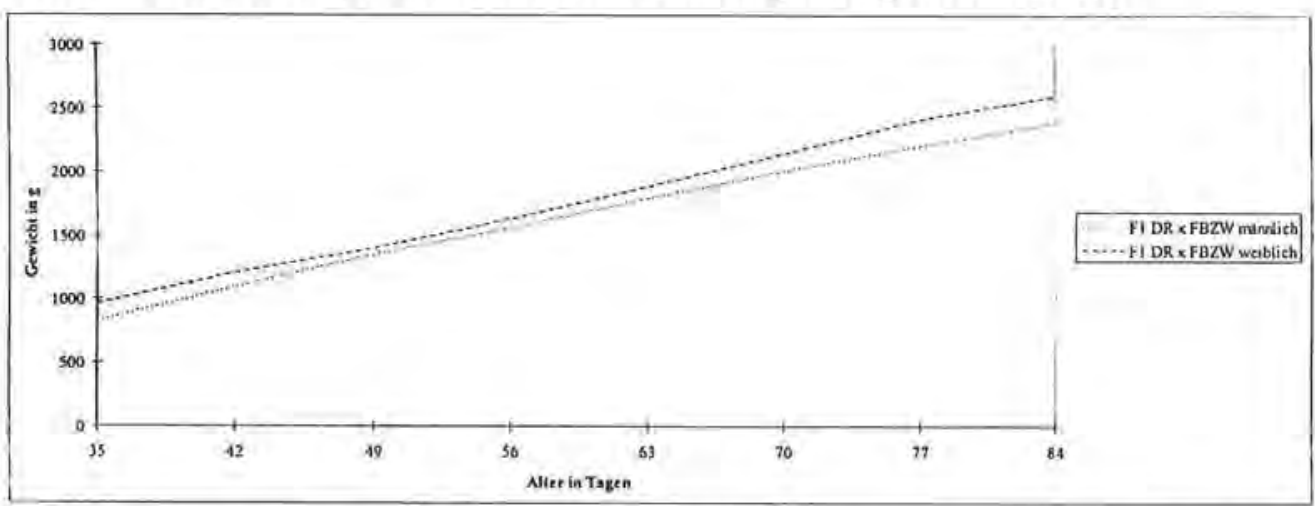

Abb. 5: Gewichtsentwicklung FI DR x FBZW in Abhăngigkeit vom Geschlecht (Development of weight F1 DR $x$ FBZW in dependence on sex)

Diese genannten Unterschiede konnten aufgrund der relativ niedrigen Datensätze statistisch nicht gesichert werden, dennoch ist eine Tendenz der Werte in diese Richtung erkennbar. Verdeutlicht wird dies bei der Zusammfassung der Daten in die Klassen 35 LT- 56 LT und 63 LT- 84 LT. Innerhalb der Klasse 63 LT - 84 LT konnten jetzt signifikante Unterschiede $(\alpha=0,05)$ zwischen männlichen und weiblichen Nachkommen der Kreuzung FBZW x DR gefunden (Tab, 5) werden.

\section{Tabelle 5}

Körpergewichte der reziproken Kreuzungen nach Bildung der Klasse 63 LT - 84 LT; Ergebnisse der Varianzanalyse (Body weights of the reciprocal crosses after formation of the classes 63th - 84th day of life; results of the analysis of variance)

\begin{tabular}{|c|c|c|c|c|c|c|c|c|}
\hline \multirow[t]{2}{*}{ FI } & $\begin{array}{l}63 \mathrm{LT}-84 \mathrm{LT} \\
\text { LSMEAN }\end{array}$ & $\begin{array}{l}\text { Std Err } \\
\text { LSMEAN }\end{array}$ & $\begin{array}{l}\operatorname{Pr}>|\mathrm{T}| \\
\mathrm{HO} 0: \mathrm{LSM}\end{array}$ & \multicolumn{5}{|c|}{$\begin{array}{l}\operatorname{Pr}>|T| \text { H0: LSMEAN(i)=LSMEAN(j) } \\
\text { LEAN }=0 \quad \mathrm{i} / \mathrm{j}\end{array}$} \\
\hline & & & & & 1 & 2 & 3 & 4 \\
\hline DR $\times$ FBZW weibl. & 2267,32143 & 65,62432 & 0,0001 & 1 & - & 0,0785 & 0,0591 & 0,9055 \\
\hline DR $\times$ FBZW männl. & 2108,34375 & 61,38593 & 0,0001 & 2 & 0,0785 & $\cdot$ & 0,8700 & 0,0537 \\
\hline FBZW x DR weibl. & 2120,43056 & 40,92395 & 0,0001 & 3 & 0,0591 & 0,8700 & - & 0,0277 \\
\hline FBZW x DR månnl. & 2257,76786 & 46,40340 & 0,0001 & 4 & 0,9055 & 0,0537 & 0,0277 & - \\
\hline
\end{tabular}

Vergleicht man die Wachstumskurven der beiden reziproken Kreuzungen getrennt nach Geschlecht miteinander, ergibt sich ein in etwa gleiches Wachstum der beiden jeweils hinsichtlich ihres Gewichtes größeren Nachkommen der Kreuzungen und des jeweils unterlegenen Geschlechtes (Abb, 6). So unterschieden sich in ihren Mittelwerten am 84. LT die männliche F1 FBZW $\times$ DR von der weiblichen F1 DR $\times$ FBZW nur um $15,4 \mathrm{~g}(0,59 \%)$ und die weibliche F1 FBZW $x$ DR von der männlichen F1 DR $\mathrm{x}$ FBZW um 47,3 g (1,97\%). Bestätigt wird diese Tendenz auch bei der Betrachtung der Ergebnisse nach Bildung der Klasse 63 LT- 84 LT (Tab. 5). Hier betragen die Unterschiede zwischen den Mittelwerten der betreffenden Geschlechter 9,6 g (0,42\%) und 
$12,1 \mathrm{~g}(0,57 \%)$. Die Ergebnisse der Varianzanalyse weisen ebenfalls auf dieses Phänomen hin.

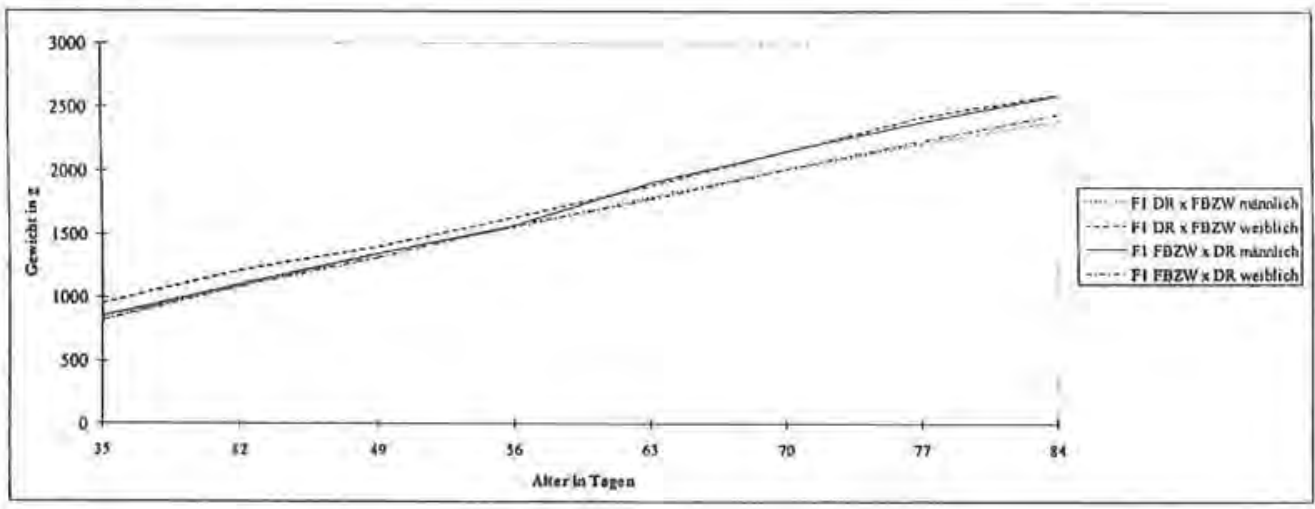

Abb. 6: Gewichtsentwicklung der reziproken F1 in Abhängigkeit vom Geschlecht (Development of weight of the reciprocal F1 in dependence on sex)

Für die Beschreibung des Zuwachses und von Zuwachsabschnitten im Verlauf der Entwicklung wurden aus den Gewichtsdaten die Zunahmen der Tiere pro Woche berechnet und in der Abbildung 7 anhand der Mittelwerte graphisch dargestellt,

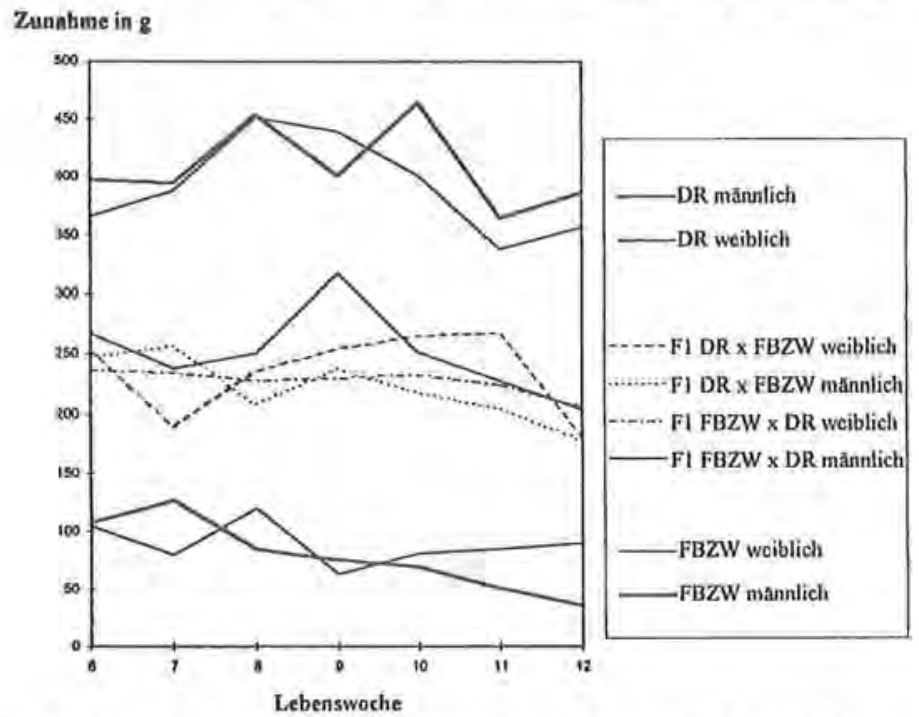

Abb. 7: Zunahume der reinen Rassen und der reziproken Kombinationen in Abhängigkeit vom Geschlecht und Lebensalter (Increase in weight of the pure-bred races and the reciprocal combinations in dependence on sex and age)

Aufgrund der gefundenen Gewichtsunterschiede zwischen den Geschlechtern der reinen Rassen sowie der Kreuzungen am 84. LT wurden die Elternrassen mit in die Betrachtung einbezogen. Hier zeigte sich, daß ab der 10, Lebenswoche (LW) höhere $\mathrm{Zu}$ wächse bei den DR wie auch bei den FBZW von demjenigen Geschlecht verwirklicht 
wurden, welches das größere Endgewicht aufwies. Innerhalb der Fl FBZW x DR übertrafen die männlichen Nachkommen die weiblichen bis zur 12. LW. Die Zunahmen der weiblichen F1 DR x FBZW stiegen in der 8. LW über die ihrer männlichen F1, nachdem diese bis zur 7. LW höhere Werte erreichten.

Die sich in der Gewichtsentwicklung gleichenden Nachkommen, männliche FBZW x DR und weibliche DR $\times$ FBZW bzw. weibliche FBZW $x$ DR und männliche DR $x$ FBZW zeigen keine übereinstimmende Zunahmekurven über die Lebenswochen. In etwa gleiche Zunahmeabschnitte, d.h. Abnahme oder Anstieg der Zunahmen bezogen auf die Lebenswochen unabhängig von der Hőhe der Zunahmen, bestehen von der 7. 9. LW bzw. von der 11. - Ende 12. LW. Ein in etwa gleicher Abschnitt besteht von der 9. - Ende 12. LW bei beiden reziproken männlichen Nachkommen.

\section{Diskussion}

Im Hinblick auf spätere Kerntransferexperimente sollte untersucht werden, ob nach dem Transfer reziproker Kernkombinationen auf eine neutrale Ammenlinie beim Kaninchen Merkmalsdifferenzen zwischen den Phänotypen bestehen, die Ausdruck von maternal zytoplasmatischen und / oder Imprinting-Effekten sein könnten. Eingangs ist darauf hinzuweisen, daß es sich aufgrund der relativ niedrigen Nachkommenzahlen bei getroffenen Aussagen bzw. der Interpretation von Ergebnissen um Tendenzen handelt.

Die gefundenen Wachstumskurven der reziproken Kreuzungen liegen zwischen denen der reinen Rassen, aber über ihrem Mittel. Dieses wird nur einmal von den Nachkommen der DR x FBZW beim Geburtsgewicht um 1,3 g unterschritten. Über die Gründe des Auftretens von solchen Heterosiseffekten bestehen unterschiedliche Theorien. Die meisten, wie Dominanztheorie, Überdominanztheorie und auch die Annahme epistatischer Effekte als Ursache des Phänomens gehen von Genen oder Genkomplexen aus. Eine weitere Möglichkeit der Begründung heterotischer Erscheinungen besteht durch Vorgänge außerhalb des Zellkernes.

Die Wurfgewichte der reziproken Kreuzungen, welche als Maßstab des pränatalen Wachstums angesehen werden können, unterscheiden sich signifikant voneinander. Die F1 DR x FBZW ist der F1 FBZW x DR um 19,25 Prozentpunkte unterlegen. Mit Ausschluß der Existenz unterschiedlicher intra-uteriner Umwelten durch den Transfer der Embryonen auf ,neutrale" Ammentiere können nur die Embryonen selbst für die gefundenen Unterschiede verantwortlich sein, da auch die Wurfgrößen sich voneinander unterscheiden (12 Prozentpunkte: F1 DR x FBZW: 5,0 Tiere/Wurf; F1 FBZW x DR: 5,6 Tiere/Wurf) und die geringeren Wurfgewichte aus kleineren Würfen stammen. Durch die Mitochondrien, die im Stoffwechsel eine gravierende Rolle spielen und neben den Prozessen der biologischen Endoxidation den Ort der Energieproduktion darstellen, ist ein Zytoplasmabestandteil vorhanden, welcher Einfluß auf die gesamten körperlichen Leistungen besitzt. Mit der mitochondrialen DNA sind die Mitochondrien in der Lage, Informationen zu speichern. Diese werden mit dem Zytoplasma rein monoparental durch die Mütter auf die Nachkommen vererbt. Im Falle der vorgestellten reziproken Kreuzungen sind Zytoplasmen von in ihrem Körpergewicht stark diverierenden Rassen (etwa 1:5) gegeben, die scheinbar für die unterschiedliche Gewichts- 
entwicklung in der pränatalen Phase verantwortlich sind.

Der geringe Gewichtsunterschied der reziproken Kreuzungen am 84. Lebenstag läßt den Schluß zu, daß der mögliche Einfluß des Zytoplasmas im Laufe der Entwicklung an Wirkung verliert und am Ende des Kontrollabschnittes nicht mehr von Bedeutung für die Gewichtsentwicklung ist.

Die Nichtexistenz von Merkmalsunterschieden hinsichtlich des Körpergewichtes am 84. Lebenstag nach Ausschluß unterschiedlicher uteriner und postnataler Bedingungen zwischen den reziproken Kreuzungsprodukten kann dahingehend interpretiert werden, $\mathrm{da} ß \mathrm{zu}$ diesem Zeitpunkt keine maternal zytoplasmatischen und / oder Imprinting-Effekte für die Gewichtsentwicklung von Bedeutung sind. Bei Auftreten dieser Effekte wären jeweils die F1-Generationen in ihrem phänotypischen Erscheinungsbild voneinander verschieden. Aus diesem Grund muß angenommen werden, daß auftretende Gewichtsunterschiede der Nachkommen reziproker Anpaarungen (ohne Transfer auf ein Ammentier), die nicht das Geburtsgewicht betreffen, ausschließlich Ausdruck der unterschiedlichen uterinen und postnatalen Umweltbedingungen sind.

Von großem Interesse dürfte das Auffinden der unterschiedlichen Gewichte von männlichen und weiblichen Tieren der reziproken Nachkommen am 84. LT sein. Die männlichen Nachkommen der Anpaarung FBZW x DR bzw, die weiblichen der Paarung DR x FBZW sind ihren weiblichen bzw. männlichen Vergleichstieren um $141,1 \mathrm{~g}$ $1203,8 \mathrm{~g}$ überlegen.

Mit 112,2 g höherem Gewicht der Farbzwerghäsinnen und $40 \mathrm{~g}$ der Riesenböcke sind die Unterschiede auch zwischen den Geschlechtern der reinen Rassen vorhanden, welche allerdings keinem Embryotransfer unterlagen. Bei den frühreifen Farbzwergen kann der Gewichtsvorteil der Häsinnen durch die Entwicklung der für die Reproduktion notwendigen Organe entstehen. Aufgrund des geringen Gewichtes der Farbzwerge ist die Differenz zwischen den männlichen und weiblichen Tieren nicht so groß, sodaß mit der Entwicklung der Reproduktionsorgane ein Gewichtsanstieg der weiblichen Tiere (zur Erlangung des für einen Wurf erforderlichen absoluten Gewichtes) verbunden ist, der über das Gewicht der Farbzwergböcke steigt. Die vom Zeitpunkt her später geschlechtsreifen Deutsche Riesen, die ihr Vollgewicht erst mit 7-8 Monaten erreichen, zeigen am 84. LT einen geringen Vorteil der männlichen Tiere. Im weiteren Wachstumsverlauf ist mit einer Vergrößerung der Differenz zu rechnen. Der mit der Entwicklung der Reproduktionsorgane verbundene Gewichtsanstieg zur Erlangung des für einen Wurf erforderlichen absoluten Gewichtes wird daher nicht über das Gewicht der männlichen Tiere ansteigen können.

Als Ursachen für die Unterschiede zwischen den jeweiligen Geschlechtern der reziproken Kreuzungen und den gleichen Wachstumskurven der reziprok verschiedenen beiden größeren bzw. kleineren Nachkommen lassen sich extrachromosomale oder auch geschlechtschromosomale Faktoren vermuten.

Für die Existenz einer extrachromosomalen bzw, zytoplasmatischen Beeinflussung (A) der unterschiedlichen Gewichtsentwicklung der Geschlechter könnte die Tatsache sprechen, daß die weiblichen Nachkommen der Kreuzung DR x FBZW und die männlichen der Kreuzung FBZW x DR die jeweils schwereren Tiere sind. Das Farbzwergzytoplasma der F1 DR x FBZW würde wie bei den reinen Farbzwergen das höhere 
HARANT u.a: Gewichtsentwicklung beim Kaninchen nach Transfer reziproker Kemkombinationen in eine neutrale Ammenlinie

Gewicht der weiblichen Nachkommen und das DR-Zytoplasma der F1 FBZW x DR würde ebenfalls wie bei den reinen Riesen das höhere Gewicht der männlichen Nachkommen bedingen. In diesem Fall muß von der Existenz von Rezeptoren im Zytoplasma ausgegangen werden, welche in der Lage sind, auf das jeweilige Geschlecht zu reagieren und ein entsprechendes Mehr- oder Minderwachstum einzuleiten.

Eine weitere Erklärung für den auftretenden reziproken Geschlechtsdimorphismus der Rassenkreuzungen könnte ein geschlechtsgebundener Erbgang (B) sein (Abb. 8). Da $\mathrm{X}$ - und Y-Chromosomen nicht homolog sind, können sie keine Allele tragen und die männlichen Tiere sind für die auf dem X-Chromosom gelegenen Genorte hemizygot. Die männlichen Nachkommen der Kreuzung DR x FBZW bzw. die der Kreuzung FBZW x DR erhalten das X-Chromosom ihrer Mütter. Aus diesem Grund zeigt sich ein geringeres Gewicht bei der männlichen F1 DR x FBZW und ein höheres bei der männlichen F1 FBZW x DR. Die beiden weiblichen F1-Generationen besitzen jeweils das X-Chromosom ihrer Mutter und das des rassenfremden Vaters. Daher müßte bei dem Zusammentreffen beider X-Chromosomen ein mendelnder Erbgang auftreten, d.h. dominante oder intermediäre Merkmalsausprägung. In beiden Fällen wäre eine von den männlichen Geschwistern zwar abweichende, aber gleiche Gewichtsentwicklung für die reziproken weiblichen Nachkommen zu erwarten, die in diesem speziellen Fall nicht eintrat. Vielmehr zeigte sich, daß die weibliche F1 DR x FBZW der weiblichen F1 FBZW x DR überlegen ist. Da die Wachstumskurven der schwereren weiblichen F1 mit der schwereren männlichen Fl und die der leichteren weiblichen F1 mit der leichteren männlichen F1 übereinstimmen (siehe Abb. 6), läßt sich schlußfolgern, daß bei Zusammentreffen verschiedener X-Chromosomen eine unterschiedliche Dominanz bezüglich der Gewichtsentwicklung auftritt. Ein solcher Dominanzwechsel (in Abhängigkeit von der Herkunft) ist Ausdruck einer geschlechtsbeeinflußten Vererbung, d.h. in Abhängigkeit vom Geschlecht erfolgt eine Dominanzregelung bei Heterozygotie. Als klassische Form ist sie für autosomale Gene bekannt und eine für beide Geschlechter zutreffende Erscheinung. Der hier auftretende Sonderfall für X-gebundene Gene impliziert, daß geschlechtsgebundene und geschlechtsbeeinflußte Vererbung gemeinsam auftreten und durch Imprinting geregelt werden.

Das jeweils dominante X-Chromosom stammt immer vom Vater und das rezessive immer von der Mutter ab. Damit wäre ein maternales Imprinting mit patroklinem Erscheinungsbild hinsichtlich des geschlechtsspezifischen Körpergewichtes bei weiblichen Tieren gegeben. Hierbei kann es sich um paternal beeinflußte Dominanz (B1) der X-Chromosomen oder um maternal beeinflußte Rezessivität (B2) der XChromosomen (extrachromosomal induziert) handeln. Beide möglichen Gründe für das Imprinting könnten auch als Komplex auftreten und gemeinsam für das unterschiedliche Dominanzverhalten der X-Chromosomen verantwortlich sein.

Dieses unterschiedliche Verhalten der X-Chromosomen würde bedeuten, daß unabhängig von der dem Zufall unterliegenden Ausschaltung eines der beiden X-Chromosomen (maternal oder paternal) in den einzelnen Körperzellen der weiblichen Tiere (Lyon-Hypothese, 1961) noch Gene gefordert sind, die herkunftsgemäße Aktivität zeigen.

Für die reinen Rassen Farbzwerge und Deutsche Riesen sind die gleichen X-Faktoren 
anzunehmen. Eine Dominanz oder Rezessivität von X-Chromosomen würde hier ohne Effekte verlaufen, was wiederum für extrachromosomale bzw. zytoplasmatische Faktoren (A) als Ursache für die Gewichtsdifferenzen sprechen würde.

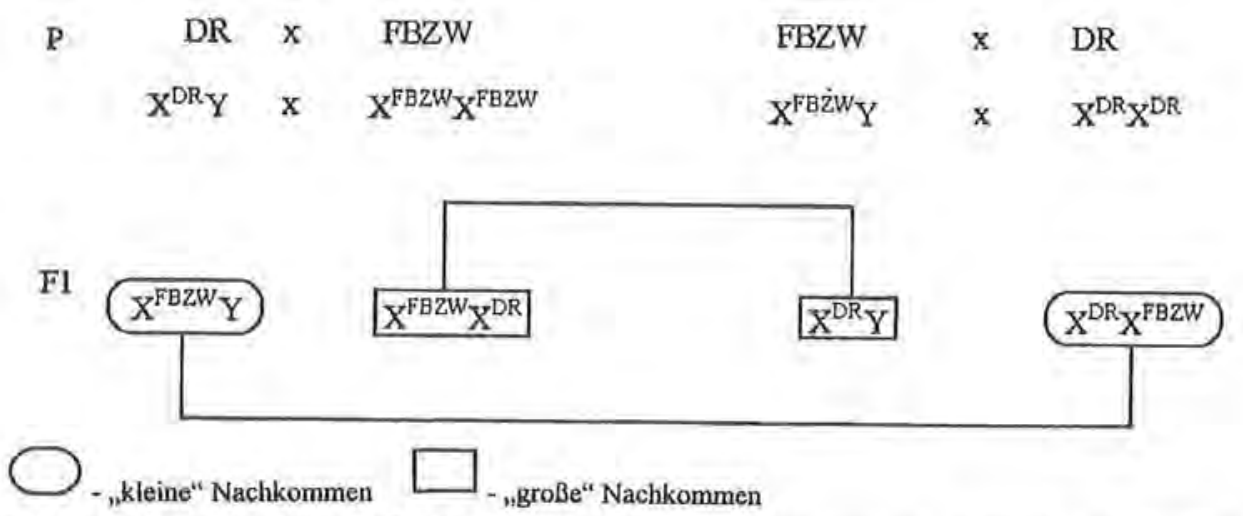

Abb. 8; Verteilung der Geschlechtschromosomen in Abhängigkeit von der Herkunft und auftretende Gewichtsunterschiede der F1-Generationen nach Transfer auf eine neutrale Ammenlinie (Allotment of the sex chromosomes in dependence on origin and differences in weight of the F1-generations after transfer to a neutral
foster line)

Bei der Annahme einer geschlechtsgebundenen Vererbung sollte auch die Möglichkeit einer Übertragung über das Y-Chromosom nicht ausgeschlossen werden. Innerhalb der reinen Rassen Deutsche Riesen und Farbzwerge wäre eine Vererbung vom Vater auf den Sohn, vom Sohn auf den Enkel usw. denkbar. Nach der reziproken Verpaarung müßten die männlichen Nachkommen der Kreuzung FBZW x DR bzw. DR x FBZW ein kleineres bzw. größeres Gewicht aufweisen. Da dies nicht der Fall ist, dürfte eine an das Y-Chromosom gebundene Vererbung nicht für den auftretenden reziproken Geschlechtsdimorphismus der Rassenkreuzungen verantwortlich sein.

Anhand von reziproken Kreuzungen der beiden Fl-Generationen, d.h. DR $\times$ FBZWBöcke mit FBZW x DR-Weibchen bzw. FBZW x DR-Böcke mit DR x FBZW-Weibchen (nachfolgend F2 genannt), sollten sich die möglichen Gründe für den reziproken Geschlechtsdimorphismus näher eingrenzen lassen.

Falls extrachromosomale Faktoren die Gewichtsunterschiede der Geschlechter bedingen, wäre das von der Parentalgeneration weitergegebene Zytoplasma in der F1- und F2-Generation ausschlaggebend (A). So müßten die männlichen Nachkommen aus der Verpaarung F1 DR $x$ FBZW mit F1 FBZW x DR und die weiblichen Nachkommen aus der Verpaarung F1 FBZW $\times$ DR mit F1 DR x FBZW ihren weiblichen und männlichen Vergleichstieren am 84. LT im Körpergewicht uberlegen sein (wie in reiner Rasse).

Die reziproken Kreuzungen der reziproken F1 würden die in Abbildung 9 dargestellten Kombinationen der weiblichen Geschlechtschromosomen ergeben, die im Falle einer geschlechtsgebundenen Vererbung (B) für die Gewichtsunterschiede verantwortlich wären. Dabei würden sich bei paternal beeinflusster Dominanz der X-Chromosomen 
(B1), wie auch bei exrachromosomal verursachter Rezessivität des mütterlichen $X$ (B2) die zu erwartenden Körpergrößen gleichen. - Derzeit vorliegende Daten von den reziprok erzeugten F2-Nachkommenschaften lassen aufgrund der noch geringen Tierzahl keine endgültige Deutung zu, weisen aber am ehesten auf die in Abbildung 9 veranschaulichte Bedeutung der X-Chromosomen hin.

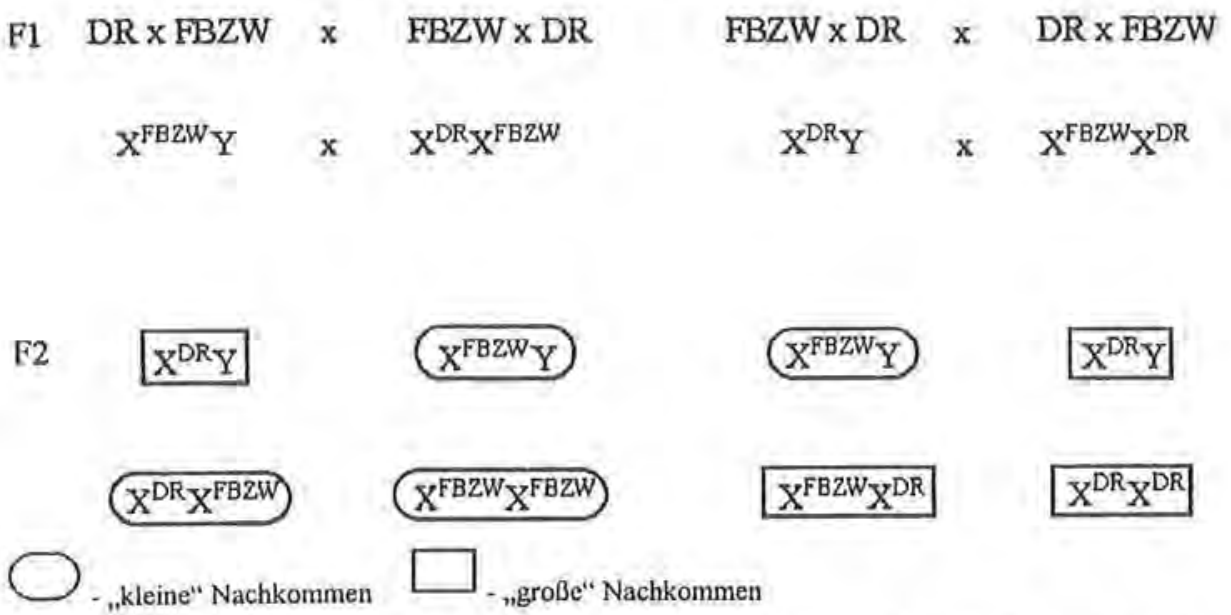

Abb. 9: Verteilung der Geschlechtschromosomen nach reziproker Kreuzung der F1 und daraus abgeleitete
Größenunterschiede (Allotment of the sex chromosomes after reciprocal cross of the F1 and derived differences
of size)

Dies hätte zur Folge, daß beide männlichen F2-Nachkommen in „größere“ bzw. „kleinere" Tiere aufspalten würden. In Abhängigkeit von der Anzahl der Nachkommen und damit der Verteilung der Spaltungsgruppen müßten sich ihre Mittelwerte gleichen. Die der weiblichen F2-Tiere müßten ein höheres Gewicht der Kreuzung F1 FBZW x DR mit F1 DR x FBZW gegenüber der Kreuzung F1 DR x FBZW mit F1 FBZW x DR zeigen. Bei der Betrachtung beider Geschlechter wären die größten Mittelwerte für die weibliche F2 (F1 FBZW x DR X F1 DR x FBZW) gefolgt von den gleichgroßen männlichen Nachkommen und der unterlegenen weiblichen F2 (F1 DR x FBZW X F1 FBZW x DR) zu erwarten. Der Vergleich beider F2-Generationen sollte ein höheres Gewicht der F2 (F1 FBZW x DR X F1 DR x FBZW) ergeben.

Mit Hilfe der reziproken Kreuzungen der beiden F1-Generationen können die möglichen Ursachen des unterschiedlichen Gewichtes der männlichen und weiblichen Nachkommen hinsichtlich extrachromosomaler und geschlechtschromosomaler Faktoren näher eingegrenzt werden. Ein eventuelles komplexes Auftreten beider genannter möglichen Gründe oder eine Bestimmung der verschiedenen X-Dominanzen sind nicht aufzeigbar. Aus diesem Grunde wäre eine einleitend angesprochene Mikromanipulation die Methode der Wahl. Die Einbringung der Kernkombination DR x FBZW in DR-Zytoplasma bzw. der Kernkombination FBZW x DR in FBZW-Zytoplasma würde bei den einzelnen vermuteten Ursachen die in Tabelle 6 dargestellten „Körpergrößen“ zur Folge haben. Anhand dieser zu erwartenden Körpergrößen und den real auftreten- 
den ließen sich genauere Rückschlüsse auf entsprechend wirkende Faktoren oder Faktorenkomplexe ziehen.

\section{Tabelle 6}

Erwartete Köpergroßen nach Transfer der Kernkombinationen in Zytoplasma der Vatertierrasse (Expected body size after transfer of the nuclear combinations into the cytoplasm of the father-race)

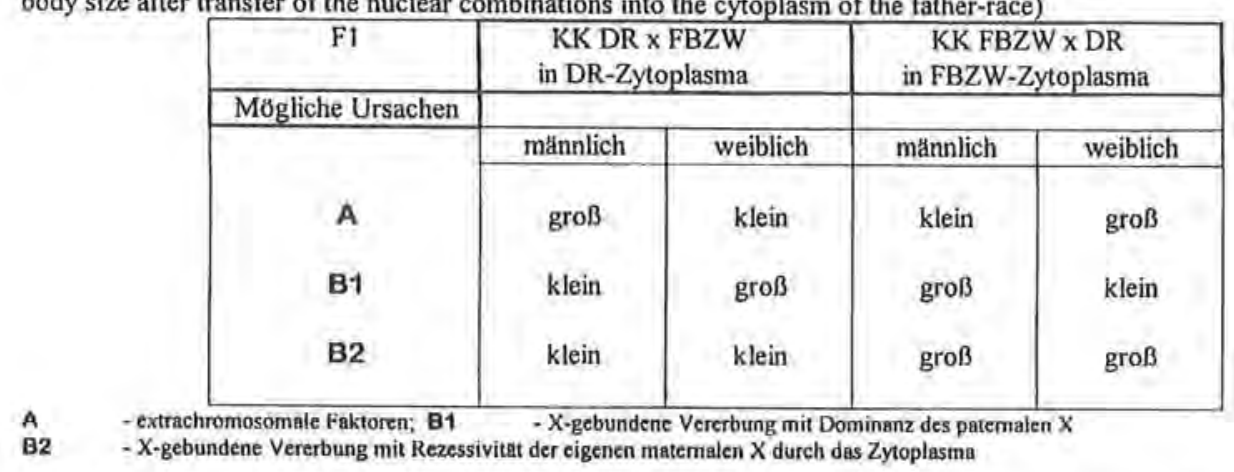

Mit der Bestimmung des mittleren Zuwachses der Elterntierrassen sowie der F1-Generationen in den Lebenswochen (LW) 6 - Ende 12 solite geklärt werden, ob im Verlauf der postnatalen Entwicklung gleiche Zuwächse und Zuwachsabschnitte (bezogen auf Anstieg und Abnahme des Zuwachses) bei den Kreuzungen und den reinen Rassen bestehen. Bei Betrachtung der Zunahmen wird deutlich, daß keine sich entsprechenden Werte über die einzelnen Lebenswochen bei den reziprok verschiedenen F1-Generationen existieren. Auch bei den sich in der Entwicklung des Körpergewichtes gleichenden Geschlechtern ist dies nicht der Fall. Es konnten jedoch einzelne Zuwachsabschnitte aufgezeigt werden, die als Zeitpunkte für das Wirken vermuteter Ursachen in der Ontogenese Bedeutung besitzen könnten. Hierbei handelt es sich um die in etwa gleichen Zuwachsabschnitte (Anstieg bzw. Abfall) bei der männlichen F1 FBZW x DR und der weiblichen F1 DR x FBZW von der 7. - 9. Lebenswoche, bei beiden männlichen Kreuzungstieren von der 9. - Ende 12. Lebenswoche und bei der männlichen F1 DR x FBZW und weiblichen F1 FBZW x DR von der 11. - Ende 12. Lebenswoche. Eine Interpretation der genannten Abschnitte kann erfolgen, indem alle aufgeführten möglichen Ursachen für das Auftreten der Gewichtsunterschiede in die Betrachtung einbezogen werden. Die gleichen Zuwachsabschnitte bei beiden männlichen Nachkommengruppen, welche Ende der 8. LW ihren Ausgangspunkt aufweisen (Peak Anfang 9. - Ende10. LW) lassen den Schluß zu, daß in diesem Zeitraum eine geschlechtsbeeinflußte Vererbung, hervorgerufen durch Geschlechtshormone zur Wirkung kommt. Nicht auszuschließen ist hier eine Wechselwirkung zwischen dem jeweiligen Geschlecht und dem Zytoplasma, welches demnach in der Lage sein müßte, dieses zu erkennen. Mit der Feststellung, daß erst ab dieser Zeit das Geschlecht von Bedeutung ist, kann man davon ausgehen, daß in der 7. - 9. LW diese Faktoren keine Rolle spielen und demnach nur die X-Chromosomen einen Einfluß besitzen.

Es ergibt sich nun die Frage, warum in dieser Zeit (7. - 9. LW) gleiche Zuwachsabschnitte bei den später größeren Nachkommen (männliche F1 FBZW x DR und weibliche F1 DR x FBZW) existieren und bei den später kleineren Nachkommen (männliche 
HARANT u.a: Gewichtsentwicklung beim Kaninehen nach Transfer reziproker Kernkombinationen in cine neutrale Ammenlinie

F1 DR $\times$ FBZW und weibliche F1 FBZW x DR) nicht. Eine Beantwortung dieser Frage scheint möglich, indem man beide angesprochenen Möglichkeiten für die Initiierung des Imprintings einbezieht, d.h. paternal beeinflußte Dominanz der XChromosomen (B1) und extrachromosomal verursachte Rezessivität der mütterlichen $X$ (B2). Die gleichen Zuwachsabschnitte bei den später größeren Nachkommen sind Ausdruck dafür, daß nur die $\mathrm{X}^{\mathrm{DR}}$-Chromosomen wirken und das $\mathrm{X}^{\mathrm{FBZW}}$-Chromosom der weiblichen Tiere paternal bedingt imprintiert wurde (B1). Bei den später kleineren Nachkommen müßten zu diesem Zeitpunkt nur die $\mathrm{X}^{\mathrm{FBZW}}$-Chromosomen wirksam sein und das $\mathrm{X}^{\mathrm{DR}}$-Chromosom dem Imprinting unterliegen, um ebenfalls gleiche Zuwachsabschnitte zu erlangen. Da dies nicht der Fall ist, müssen beide weiblichen XChromosomen noch aktiv sein. Das Imprinting der $\mathrm{X}^{\mathrm{DR}}$-Chromosomen erfolgt hier wahrscheinlich erst von der 9. - 11. Lebenswoche unter dem Einfluß des Geschlechtes und des Zytoplasmas (B2), wie für diese Zeit bei beiden männlichen Nachkommengruppen vermutet wurde. Mit dem nun vollzogenem Imprinting sind nur noch die $\mathrm{X}^{\mathrm{FB} 2 \mathrm{~W}}$-Chromosomen aktiv und dadurch fur die gleichen Wachstumsabschnitte von der 11, - Ende 12. LW verantwortlich. Die später größeren Nachkommen unterliegen ebenso in der 9. - 11. LW dem Einfluß des Geschlechtes und des Zytoplasmas. Die in dieser Zeit angenommene Reaktion des Zytoplasmas auf das Geschlecht sollte bewirken, daß das jeweilige Geschlecht in dieser Zeit einen Vorteil gegenüber dem anderen bekommt. Da die später größeren Nachkommen auch als größere von ihrem Zytoplasma erkannt werden, erfolgt auch diese Reaktion. Der Unterschied besteht aber in den verschiedenen zytoplasmatischen Umwelten, wodurch ein ungleiches „Potential" (DR- bzw. FBZW-Zytoplasma) vorhanden ist und damit auch ungleiche Zuwachsabschnitte bewirkt werden. Ein Hinweis für die Erkennung des Geschlechtes durch das Zytoplasma ist bei der F1 FBZW x DR gegeben. Das Gewicht der männlichen Tiere steigt in der 9. LW über das der weiblichen Nachkommen. Bei den später kleineren Nachkommen findet die Reaktion des Zytoplasmas nicht statt, da sie von ihm als die jeweils kleineren Geschlechter erkannt werden und somit die Verschaffung eines Vorteils ausgeschlossen wird.

Eine genaue Aufdeckung und Deutung weiterer Abschnitte ist aus Gründen der ungeklärten physiologischen Vergleichbarkeit der reinen Rassen untereinander und mit den Kreuzungen nur bedingt möglich und soll hier nicht näher betrachtet werden.

Zusammenfassend kann man sagen, daß für die unterschiedlichen Gewichtsentwicklungen der Geschlechter der reziproken Kreuzungen aus Deutschen Riesen und Farbzwergen nach Transfer auf ein neutrales Ammentier vermutlich der Sonderfall einer geschlechtsgebundenen und geschlechtsbeeinflußten Vererbung verantwortlich ist. Dieser scheint durch zytoplasmatische- wie auch Imprinting-Effekte, die im Komplex wirken, geregelt zu werden. Eine Ausnahme dieser komplexen Wirkung besteht in der pränatalen Phase, wo nur das Zytoplasma verantwortlich scheint. Eine genaue Klärung der Ursachen für die auftretenden Gewichtsunterschiede dürfte nur mit Hilfe einer Mikromanipulation und damit der Einbringung der Kernkombination DR $\times$ FBZW in DR-Zytoplasma bzw. der Kernkombination FBZW x DR in FBZW-Zytoplasma möglich sein. 


\section{Literatur}

CHANDRA, H.S,; BROWN, S.W.:

Chromosome imprinting and the mammalian X-chromosome. Nature, London 253 (1975), 165-168

COOPER, D.W.; VANDEBERG, J.L.; SHARMA, G.B.; POOLE, W.E.: Phosphoglycerate kinase polymorphism in kangaroos provides further evidence for paternal $\mathrm{X}$ inactivation. Nature, London 230 (1971), 155-157

DAWID, I.B; BLACKLER, A.W:

Maternal and cytoplasmic inheritance of mitochondrial DNA in Xenopus. Devel. Biol,, 29 (1972), $152-$ 161

DE GEORGI, C.; SACCONE, C.:

Mitochondrial genom in animal cells, Cell Bio-physics, 14 (1989), 67-78

FLAVELL, R.B.; O'DELL, M.:

Variation and inheritance of cytosine methylation patterns in wheat at the high molecular weight

HAFEZ, E.S.E.:

glutenin and ribosomal RNA gene loci. Development, 1990, Suppl., 15-20

Symposium on growth: Physio-genetics of prenatal and postnatal growth. J. Anim. Sci., 22 (1963), 779

HARING, F,; STEINBACH, J.; SCHEVEN, B:

Untersuchungen uber den mitterlichen Einfluß auf die prä- und post-natale Entwicklung von Schweinen extrem unterschiedlicher Große. I. Wachstumsstudien an reziproken Bastarden von Riese (Deutsches veredeltes Landschwein) und Zwerg (Vietnamese) im Vergleich zu Schweinen der Ausgangspopulation. Z. Tierzalchtung und Zachtungsbiologie, 82 (1966), 37-53

HESLOP-HARRISON, J.S.:

Gene expression and parental dominance in hybrid plants. Development, 1990, Suppl., 21-28

HUGHES-SCHRADER, S.:

Cytology of coccids (Coccoidea-Homoptera). Adv. Genet, 2 (1948), 127-203

HUGHES-SCHRADER, S.:

The chromosome cycle of Phenacoccus (Coccidea), Biol. Bull. mar. biol, Lab., 1935, Woods Hole 69, $462-468$

HUTCHISON, C.A.; NEWBOLD, J.A.; POTTER, S.S.; EDGELL, M.H.: Matemal inheritance of mammalien mitochondrial DNA. Nature, London 251 (1974), 536-538

JOHANSSON, I.; VENGE, O.:

A study of the variation in weight during growth, and in some skeletal characteristics of adult rabbits Kungl. Lantbrukshøgskolans Annaler, 19 (1953), 161-195

JOUBERT, D.M:; HAMMOND, J.:

Maternal effect on birth weight in South Devon X Dexter Cattle crosses. Nature, London 174 (1954). 647-648

KERMICLE, J.L;; ALLEMAN, M:

KLAR, A.J.S.;

Gametic imprinting in maize in relation to the angiosperm life cycle. Development, 1990, Suppl., 9-14

Regulation of fission yeast mating-type interconversion by chromosome imprinting. Development, 1990,

LYON, M.F.: Suppl., 3-8

Gene action in the X-chromosome of the mouse (Mus musculus L.). Nature, London 190 (1961), 372. 373

NUR, U.:

Heterochromatization and euchromatization of whole genomes in scale insects (Coccoidea Homoptera).

Development, 1990, Suppl., 29-34

RICHARDSON, B.J.; CZUPPON, A.B.; SHARMAN, G.B.:

Inheritance of glucose-6-phosphate dehydrogenase variation in kangaroos. Nature, London 230 (1971), 154-155

SCHRADER, F; HUGHES-SCHRADER, S:

SCHRADER, F.: Haploidy in metazoa. Q. Rev. Biol., 1931, 6, 411-438

STENGEL, H.:

The chromosomes of Pseudococcus nipea. Biol. Bull. mar. biol. Lab., 1921, Woods Hole 40, 259-270

Fortpflanzungsbiologische Probleme der extremen Rassenkreuzung, Z. Tierzilchtung und Zuchtungsbiologie, 77 (1962), 365-373 
TAKAGI, N., SASAKI, M:

Preferential inactivation of the paternally derived X-chromosome in the extra-embryonic membranes of the mouse, Nature, London 256 (1975), 640-642

VENGE, $\mathrm{O}$ :

Studies of the maternal influence on the birth weight in Rabbits. Acta Zoologica, 1950, Bd. XXXI, 1148

VENGE, O.:

Studies of the maternal influence on the growth in rabbits. Acta Agriculturae Scandinavica, 1953, Vol. III, 243-291

WALTON, A.; HAMMOND, J:

The matemal effects on growth and conformation in Shire horse-Shetland pony crosses. Proceedings of the Royal Society of London, 1938, Vol, CXXV, 31 I-335

WEHNER, R.; GERING, W::

Zoologie. Georg Thieme Verlag Stuttgart - New York, 1990, I52-I53

WOLSTENHOLME: zit, n. JÄNKE, A:

Struktur, Funktion und Evolution des mitochondrialen Genoms der Săugetiere. München Uni, Diss., 1994

Eingegangen: 30.10 .1998

Akzeptiert: 13.12.1999

Anschrif der Verfasser

Dr. HENDRIK HARANT, Dr. agr. RALF PORZIG, DVM JENS THIELEBEIN,

Dr. rer. nat. WERNER SAAR, Prof. Dr. habil, LUTZ SCHÜLER

Landwirtschaftliche Fakultăt der Martin-Luther-Universität Halle-Wittenberg

Institut fur Tierzucht und Tierhaltung mit Tierklinik

Adam-Kuckhoff-Str. 35

D-6108 Halle 